\title{
Torsade de pointes in initiating hemodialysis: a case report
}

\author{
Ji Ae Yang, Hong Sang Choi, Eun Hui Bae, Seong Kwon Ma, Soo Wan Kim, Chang Seong Kim \\ Department of Internal Medicine, Chonnam National University Medical School, Gwangju, Korea \\ Correspondence to: Chang Seong Kim, MD, PhD. Department of Internal Medicine, Chonnam National University Medical School, 42 Jebongro, \\ Gwangju 61469, Korea. Email: laminion@hanmail.net.
}

\begin{abstract}
Prolongation of the QT interval by antiarrhythmic drugs is the primary cause of torsade de pointes. Although there are previous reports of drug-induced torsade de pointes in patients undergoing hemodialysis, torsade de pointes caused by a sudden decrease of potassium levels in patients initiating hemodialysis has not been well described. A 70-year-old woman with recurrent bilateral gluteal abscesses visited the hospital for antibiotic treatment. Twenty-eight days after admission, atrial fibrillation with rapid ventricular rhythm was newly detected and was controlled with intravenous amiodarone treatment. After developing pulmonary edema that did not improve with diuretic treatment, she underwent emergency hemodialysis. During hemodialysis, serum potassium and magnesium levels decreased to 3.1 and $1.7 \mathrm{mg} / \mathrm{dL}$, respectively. The electrocardiogram showed torsade de pointes. Amiodarone treatment was stopped, and magnesium sulfate was infused. A higher concentration of potassium dialysate was used in continuous renal replacement therapy. Torsade de pointes episodes halted, and QT interval prolongation normalized. We describe a case with a rare complication of torsade de pointes upon initiating hemodialysis in a patient with QT prolongation. When initiating hemodialysis, serum potassium levels as well as electrocardiograms should be monitored in patients with a prolonged QT interval.
\end{abstract}

Keywords: Long QT syndrome; hemodialysis; torsade de pointes; case report

Submitted Oct 21, 2019. Accepted for publication Mar 04, 2020.

doi: 10.21037/apm.2020.04.29

View this article at: http://dx.doi.org/10.21037/apm.2020.04.29

\section{Introduction}

Acquired long QT syndrome can be caused by certain medications, such as Class III and Ia antiarrhythmics, antipsychotics, tricyclic antidepressants, macrolide antibiotics, and methadone (1-3). Prolongation of the QT interval itself in a population with long QT syndrome is clinically important because prolonged QT interval is a prerequisite for torsade de pointes, an uncommon and potentially fatal type of ventricular tachycardia (4). The previously listed drugs slow down the rate of repolarization that can result in torsade de pointes. Torsade de pointes preferentially occurs with certain predisposing conditions, including heart disease, bradycardia, and electrolyte disturbances such as hypokalemia and hypomagnesemia $(1,5)$.

In dialysis patients, potassium excretion is achieved by diffusion and convection of potassium from a higher concentration in serum to a lower concentration in dialysate, and, thus, is directly proportional to the concentration gradient between serum and dialysate. Several studies have associated low dialysate potassium with a higher risk of sudden cardiac death (6-9). Karnik et al. showed that patients who suffered cardiac arrest were more likely to have been treated with a low potassium dialysate on the day of cardiac arrest (6). Moreover, Pun et al. reported that a potassium dialysate $\leq 2 \mathrm{mEq} / \mathrm{L}$ doubled the risk of sudden cardiac arrest from $10 \%$ to $20 \%$ compared to a higher potassium dialysate $\geq 2 \mathrm{mEq} / \mathrm{L}$, regardless of predialysis serum potassium levels (8). A cross-over single blind study also showed in a subset of dialysis-sensitive patients that treatment using a constant potassium dialysate with a relatively low potassium concentration $(2.5 \mathrm{mEq} / \mathrm{L}) \mathrm{K}$ resulted in a 3.9-times higher incidence of arrhythmia than treatment using decreasing intra-dialysis potassium with higher 


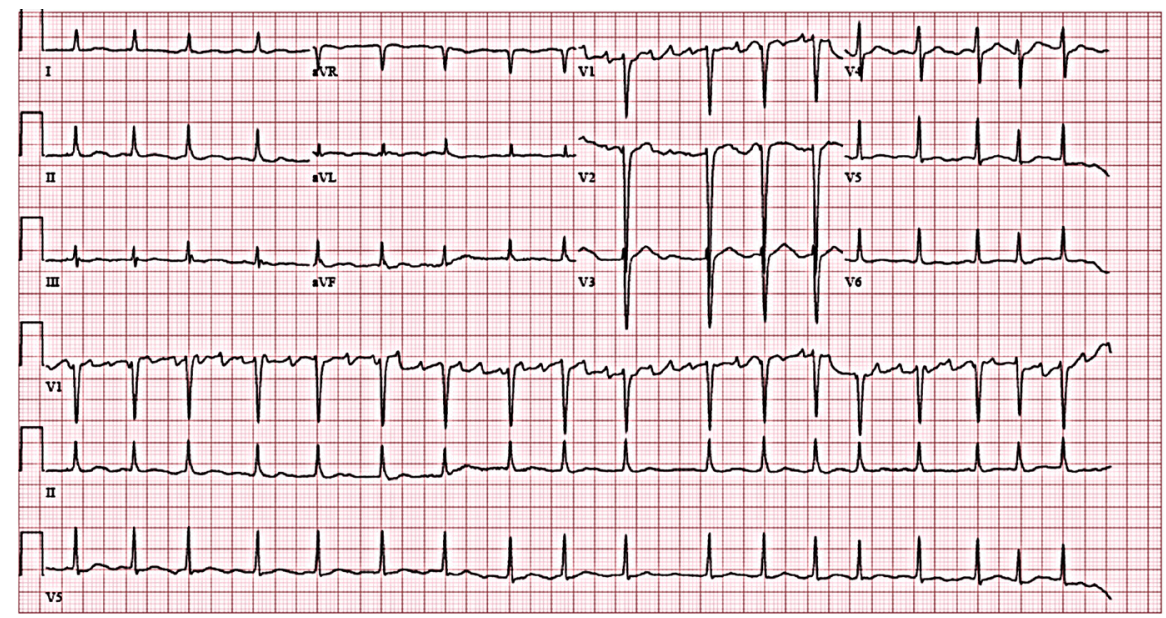

Figure 1 Electrocardiogram showing the atrial fibrillation with rapid ventricular response and corrected QT interval was 486 msec.

serum potassium concentration (10). In addition, hypokalemia is a common complication during hemodialysis (11). In this respect, hemodialysis itself might be a risk factor for prolongation of the QT interval and torsade de pointes.

To our knowledge, there have been reports of drug-induced torsade de pointes in patients undergoing hemodialysis (12-15). However, cases of torsade de pointes caused by sudden decreases of potassium levels in patients initiating hemodialysis have not been well described. We report this case of torsade de pointes occurring upon initiating hemodialysis on a patient with atrial fibrillation accompanying QT prolongation after treatment with an antiarrhythmic agent.

We present the following article/case in accordance with the CARE Guideline. A completed CARE reporting checklist (available at http://dx.doi.org/10.21037/ apm.2020.04.29).

\section{Case presentation}

A 70-year-old woman without arrhythmia or cardiac disease other than chronic kidney disease, diabetes mellitus and hypertension was admitted to the emergency room due to recurrent buttock abscesses for antibiotics treatment. Twenty-eight days after admission, atrial fibrillation with rapid ventricular rhythm was newly detected, but her heart rate was controlled $(<110$ beats/min) two days after administration of intravenous amiodarone (Figure 1). Although serum blood urea nitrogen and creatinine were markedly increased (74.9 and $5.42 \mathrm{mg} / \mathrm{dL}$, respectively), serum potassium was within the normal range $(3.8 \mathrm{mg} / \mathrm{dL})$. However, pulmonary edema was triggered despite using furosemide; thus, she received hemodialysis. After three sessions of hemodialysis, she complained of dizziness and showed a decrease in consciousness. The electrocardiogram showed polymorphic ventricular tachycardia with prolonged corrected QT interval (QT/ $\sqrt{\mathrm{RR}}$ interval) of $560 \mathrm{~ms}$, followed by torsade de pointes (Figure 2). Her serum potassium had decreased to $3.1 \mathrm{mg} / \mathrm{dL}$ (reference range $3.5-5.1 \mathrm{mEq} / \mathrm{L}$ ), total calcium to $8.7 \mathrm{mg} / \mathrm{dL}$ (reference range $8.4-10.2 \mathrm{mg} / \mathrm{dL}$ ), and magnesium to $1.7 \mathrm{mg} / \mathrm{dL}$ (reference range 1.9-2.5 mg/dL). We performed directcurrent cardioversion-defibrillation three times to correct the torsade de pointes. We discontinued amiodarone and administered magnesium sulfate. Afterwards, the patient was transferred to the intensive care unit and underwent continuous renal replacement therapy using potassiumand phosphate-containing dialysate for the prevention of hypokalemia. Consequently, serum potassium and magnesium increased to 4.8 and $2.5 \mathrm{mg} / \mathrm{dL}$, respectively, and the electrocardiogram showed a decreased corrected QT interval of $412 \mathrm{~ms}$. Her mental status recovered, and she had no further episodes of torsade de pointes. She was discharged with an oral beta-blocker (bisoprolol, $2.5 \mathrm{mg}$ twice a day) prescribed to control the atrial fibrillation. A follow-up electrocardiogram, conducted at the outpatient clinic a month later, showed atrial fibrillation, with a heart rate of 78 beats/min and a corrected QT interval of $454 \mathrm{~ms}$. The Clinical course of our patient were depicted in Figure 3.

\section{Discussion}

Torsade de pointes is an abnormal heart rhythm that can 


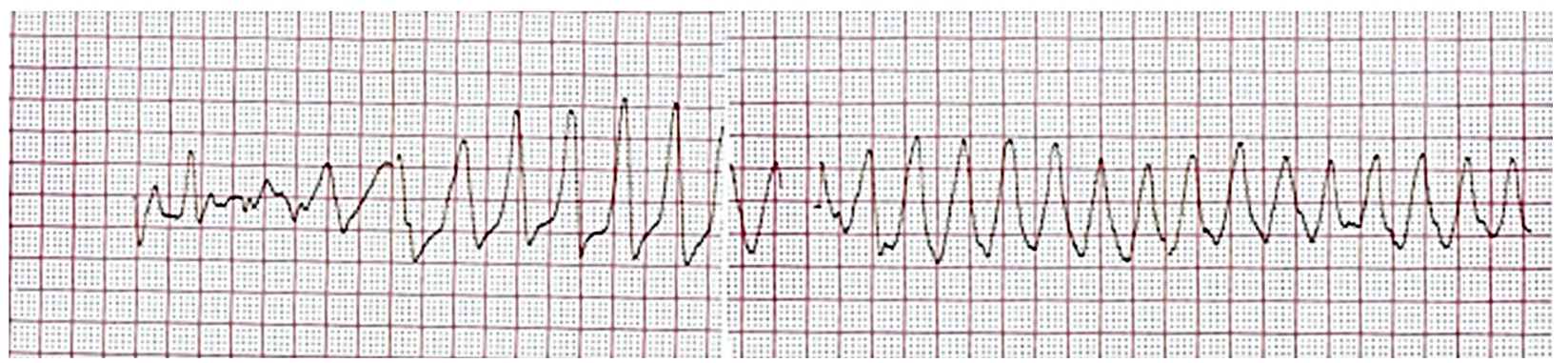

Figure 2 Electrocardiogram showing the atrial fibrillation with rapid ventricular response and corrected QT interval was 486 msec.

$$
\rightarrow-\operatorname{Serum~K}(\mathrm{mEq} / \mathrm{L}) \quad-\text { Serum } \mathrm{Mg}(\mathrm{mg} / \mathrm{dL})
$$

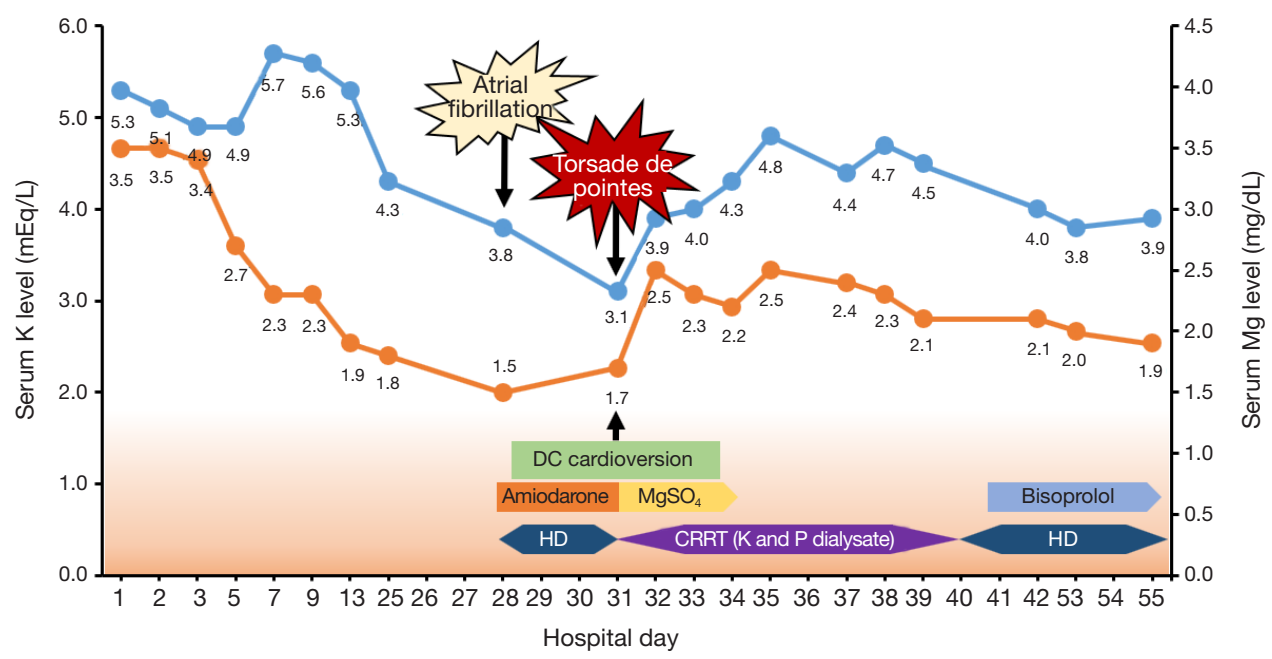

Figure 3 Clinical course of the patient. K, potassium; Mg, magnesium; P, phosphate; MgSO4, magnesium sulfate; HD, hemodialysis; CRRT, continuous renal replacement therapy.

lead to sudden cardiac death, and, thus, requires emergent treatment. Electrocardiogram shows polymorphic ventricular tachycardia and the distinct appearance of a prolonged QT interval. Early after depolarization is caused by abnormal depolarization of the potassium or sodium channels and is the triggering event for torsade de pointes (16). Although the direct underlying pathophysiology of torsade de pointes is an increased actual QT interval, predisposing factors such as female sex; electrolyte disturbances, including hypokalemia, hypomagnesemia, and hypocalcemia; medications that prolong QT interval; congestive heart failure; myocardial infarction; hypothyroidism; cerebral ischemic stroke or intracranial hemorrhage; congenital long QT syndrome; and $\mathrm{T}$ wave variability are associated with torsade de pointes $(1-3,17)$.

In our case, the prolongation of corrected QT interval might have been provoked by amiodarone treatment for atrial fibrillation in a patient with female sex as a risk factor. Although amiodarone is a well-known cause of QT prolongation and torsade de pointes, hypokalemia and hypomagnesemia brought on by hemodialysis might also trigger torsade de pointes. Consequently, physicians should check for hypokalemia when initiating hemodialysis and maintain a heart rate $\geq 70$ beats/min and a corrected QT interval of no more than $440 \mathrm{~ms}$ (18). Actually, in a prospective cohort study conducted in patients with chronic kidney disease, patients with potassium levels $\leq 4 \mathrm{mEq} / \mathrm{L}$ had a higher risk for mortality and end-stage renal disease compared to those with normal $(4.1-5.5 \mathrm{mEq} / \mathrm{L})$ or elevated (5.5-5.9 $\mathrm{mEq} / \mathrm{L})$ potassium levels (19).

Maintaining serum electrolytes at appropriate levels during hemodialysis is key to avoiding torsade de pointes. It 
is important to prevent a sharp decline in serum potassium concentration during hemodialysis because of the risk of arrhythmia. Magnesium levels also show a J-shaped curve association with all-cause mortality, especially hypomagnesemia associated with QT dispersion (20). Indeed, for hemodialysis patients with accompanying torsade de pointes, previous studies have shown that the immediate infusion of intravenous magnesium sulfate and potassium is the first treatment option $(2,18)$.

Regrettably, we could not perform a laboratory test during three consecutive dialysis session, except for the first start of dialysis. Nevertheless, a remarkable finding of our case was that the dialysate containing a physiologic level of potassium was used to treat torsade de pointes. Conventional hemodialysis, especially continuous renal replacement therapy, uses a dialysate with little to no potassium. This conventional approach could induce severe hypokalemia (21). Therefore, a potassium- and phosphatecontaining dialysate may be required in patients who are vulnerable to prolongation of QT interval to prevent severe hypokalemia.

In conclusion, hemodialysis could be a risk factor for torsade de pointes among patients with prolonged QT interval. Thus, early recognition and prevention of torsade de pointes are critical to patients with risk factors for prolonged QT interval and may help reduce morbidity and mortality. Therefore, serum potassium levels and electrocardiograms should be monitored in those patients with extended QT intervals when initiating hemodialysis.

\section{Acknowledgements}

Funding: This work was supported by the National Research Foundation of Korea (NRF) grant funded by the Korea government (MSIT) (NRF- 2017M3A9E8023018, 2017M3A9E8023001), by Basic Science Research Program through the NRF funded by the Ministry of Education (NRF-2018R1D1A1B07042999), and by a grant (BCRI18025 and BCRI20062) Chonnam National University Hospital Biomedical Research Institute.

\section{Footnote}

Reporting Checklist: The authors have completed the CARE reporting checklist. Available at http://dx.doi.org/10.21037/ apm.2020.04.29

Conflicts of interest: All authors have completed the ICMJE uniform disclosure form (available at http://dx.doi. org/10.21037/apm.2020.04.29). The authors have no conflicts of interest to declare.

Ethical Statement: The authors are accountable for all aspects of the work in ensuring that questions related to the accuracy or integrity of any part of the work are appropriately investigated and resolved. The authors obtained approval from the institutional review board (IRB) of Chonnam National University Hospital (CNUH2019-204), and informed consent was waived by the IRB. Written informed consent was obtained from the patient for publication of this case report and any accompanying images.

Open Access Statement: This is an Open Access article distributed in accordance with the Creative Commons Attribution-NonCommercial-NoDerivs 4.0 International License (CC BY-NC-ND 4.0), which permits the noncommercial replication and distribution of the article with the strict proviso that no changes or edits are made and the original work is properly cited (including links to both the formal publication through the relevant DOI and the license). See: https://creativecommons.org/licenses/by-nc-nd/4.0/.

\section{References}

1. Bahrle S, Schols W. Torsade de pointes in haemodialysis patients. Nephrol Dial Transplant 1996;11:944-6.

2. Uvelin A, Pejakovic J, Mijatovic V. Acquired prolongation of QT interval as a risk factor for torsade de pointes ventricular tachycardia: a narrative review for the anesthesiologist and intensivist. J Anesth 2017;31:413-23.

3. Schwartz PJ, Woosley RL. Predicting the Unpredictable: Drug-Induced QT Prolongation and Torsades de Pointes. J Am Coll Cardiol 2016;67:1639-50.

4. Gupta A, Lawrence AT, Krishnan K, et al. Current concepts in the mechanisms and management of druginduced QT prolongation and torsade de pointes. Am Heart J 2007;153:891-9.

5. el-Sherif N, Zeiler RH, Craelius W, et al. QTU prolongation and polymorphic ventricular tachyarrhythmias due to bradycardia-dependent early afterdepolarizations. Afterdepolarizations and ventricular arrhythmias. Circ Res 1988;63:286-305.

6. Karnik JA, Young BS, Lew NL, et al. Cardiac arrest and sudden death in dialysis units. Kidney Int 2001;60:350-7.

7. Pun PH, Goldstein BA, Gallis JA, et al. Serum Potassium 
Levels and Risk of Sudden Cardiac Death Among Patients With Chronic Kidney Disease and Significant Coronary Artery Disease. Kidney Int Rep 2017;2:1122-31.

8. Pun PH, Lehrich RW, Honeycutt EF, et al. Modifiable risk factors associated with sudden cardiac arrest within hemodialysis clinics. Kidney Int 2011;79:218-27.

9. Redaelli B, Locatelli F, Limido D, et al. Effect of a new model of hemodialysis potassium removal on the control of ventricular arrhythmias. Kidney Int 1996;50:609-17.

10. Santoro A, Mancini E, London G, et al. Patients with complex arrhythmias during and after haemodialysis suffer from different regimens of potassium removal. Nephrol Dial Transplant 2008;23:1415-21.

11. Hung AM, Hakim RM. Dialysate and serum potassium in hemodialysis. Am J Kidney Dis 2015;66:125-32.

12. Daya SK, Gowda RM, Khan IA. Ciprofloxacin- and hypocalcemia-induced torsade de pointes triggered by hemodialysis. Am J Ther 2004;11:77-9.

13. Rizza C, Valderrabano M, Singh BN. Recurrent Torsades de Pointes After Sotalol Therapy for Symptomatic Paroxysmal Atrial Fibrillation in a Patient with End-Stage Renal Disease. J Cardiovasc Pharmacol Ther 1999;4:129-34.

14. Tang S, Lo CY, Lo WK, et al. Sotalol-induced Torsade de pointes in a CAPD patient--successful treatment with intermittent peritoneal dialysis. Perit Dial Int

Cite this article as: Yang JA, Choi HS, Bae EH, Ma SK, Kim SW, Kim CS. Torsade de pointes in initiating hemodialysis: a case report. Ann Palliat Med 2020;9(3):1264-1268. doi: 10.21037/ apm.2020.04.29
1997;17:207-8.

15. Huynh-Do U, Wahl C, Sulzer M, et al. Torsades de pointes during low-dosage sotalol therapy in haemodialysis patients. Nephrol Dial Transplant 1996;11:1153-4.

16. Weiss JN, Qu Z, Shivkumar K. Electrophysiology of Hypokalemia and Hyperkalemia. Circ Arrhythm Electrophysiol 2017. doi: 10.1161/CIRCEP.116.004667.

17. Viskin S. Long QT syndromes and torsade de pointes. Lancet 1999;354:1625-33.

18. Gregoratos G, Cheitlin MD, Conill A, et al. ACC/ AHA guidelines for implantation of cardiac pacemakers and antiarrhythmia devices: a report of the American College of Cardiology/American Heart Association Task Force on Practice Guidelines (Committee on Pacemaker Implantation). J Am Coll Cardiol 1998;31:1175-209.

19. Korgaonkar S, Tilea A, Gillespie BW, et al. Serum potassium and outcomes in CKD: insights from the RRICKD cohort study. Clin J Am Soc Nephrol 2010;5:762-9.

20. Sakaguchi Y, Fujii N, Shoji T, et al. Hypomagnesemia is a significant predictor of cardiovascular and noncardiovascular mortality in patients undergoing hemodialysis. Kidney Int 2014;85:174-81.

21. Besnard N, Serveaux M, Machado S, et al. ElectrolytesEnriched Hemodiafiltration Solutions for Continuous Renal Replacement Therapy in Acute Kidney Injury: A Crossover Study. Blood Purif 2016;42:18-26. 